\title{
Incorporation de tourteau de colza obtenu par pression dans une ration d'engraissement chez le taurillon. II. Les glucosinolates et leurs dérivés dans le rumen
}

\author{
JP Wathelet ${ }^{1}$, L Istasse ${ }^{2}$, AP Mayombo ${ }^{2 *}$, M Marlier ${ }^{1}$ \\ I UER chimie générale et organique, faculté des sciences agronomiques, 5030 Gembloux ; \\ ${ }^{2}$ Nutriton animale, faculté de médecine vétérinaire, université de Liège, \\ B 43, Sart Tilman, 4000 Liège, Belgique
}

(Reçu le 27 octobre 1995 ; accepté le 12 juin 1996)

\begin{abstract}
Summary - Incorporation of rapeseed meal extracted by pressure in a diet for growing fattening bulls. II. Glucosinolates and their breakdown products in the rumen. Eight bulls from the Belgian Blue breed were offered diets in which soya bean meal and linseed meal were substituted by rapeseed meal (variety Samourai) in order to provide, respectively, 33, 66 and $100 \%$ of the protein. The glucosinolate content was equal to $17.6 \mu \mathrm{mol} / \mathrm{g}$ dry matter. Analyses of wet residues from rapeseed meal incubated in the rumen showed a rapid decrease of the glucosinolate content which disappeared after $5 \mathrm{~h}$ of incubation. The 3-hydroxypent-4-enenitrile, the but-3-enylisothiocyanate and the 5-vinyl-1,3-oxazolidine-2-thione antinutritional factors obtained from the glucosinolates were identified by GC-MS during the first $5 \mathrm{~h}$. The 3-phenylpropanenitrile, the 3-methylindol, the 2-phenylacetic and 3-phenylpropanoic acids were also present in the residues and in the rumen. Although high sensitivity of the methods were used, no glucosinolates and their direct breakdown products were detected in the liquid and the solid content of the rumen.
\end{abstract}

\section{bull / rapeseed meal / glucosinolate}

Résumé - Huit taurillons Blanc bleu belge munis d'une canule du rumen ont reçu des rations d'engraissement dans lesquelles les matières azotées des tourteaux de soja et de lin ont été remplacées respectivement par 33, 66 et $100 \%$ de matières azotées de tourteau de colza « Samourai double 00 ». Le tourteau de colza contenait 17,6 $\mu \mathrm{mol}$ de glucosinolates par gramme de MS. L'analyse des rési-

* Correspondance et tirés à part

Fax : (32) 43664122 ; courriel : Mayombo@stat.fmv.ulg.ac.be 
dus humides de tourteau de colza incubé en sachets de nylon dans le rumen montre que les glucosinolates diminuent rapidement pour disparaître après 5 heures d'incubation. Le 3-hydroxypent-4-ènenitrile, le but-3-ènylisothiocyanate et la 5-vinyl-1,3-oxazolidine-2-thione, facteurs antinutritionnels dérivant des glucosinolates, ont été mis également en évidence par chromatographie en phase gazeuse couplée à la spectrométrie de masse (GC-MS) pendant les cinq premières heures. Le 3-phénylpropanènitrile, le 3-méthylindole et les acides 2-phényléthanoïque et 3-phénylpropanoïque ont aussi été identifiés dans les résidus, le liquide et le contenu du rumen. Malgré la sensibilité des méthodes utilisées, aucun glucosinolate ou produit direct de dégradation n'a pu être détecté dans les liquides et contenus du rumen.

\section{taurillon / tourteau de colza / glucosinolate}

\section{INTRODUCTION}

La qualité nutritionnelle du tourteau de colza, source importante de protéines pour l'alimentation animale, a augmenté de manière sènsible avec l'apparition de variétés à faible teneur en glucosinolates c'est-à-dire des teneurs comprises entre 20 et $35 \mu \mathrm{mol} / \mathrm{g}$ de matière sèche (MS ; colza OO). Les possibilités d'utilisation de ces variétés sont de plus en plus accrues tant chez les polygastriques que les monogastriques (Évrard, 1991).

Les effets néfastes du tourteau de colza chez les animaux sont dus principalement aux produits de dégradation des glucosinolates. Ce sont par exemple les isothiocyanates responsables de l'inappétence et la 5-vinyl-1,3-oxazolidine-2thione responsable du disfonctionnement thyroïdien (Astwood et al, 1949 ; Greer et Deeney, 1959 ; Fenwick et al, 1986 ; Darroch et al, 1990). Cette dégradation est réalisée par une enzyme, la myrosinase, qui peut être activée pendant la trituration ou produite par la flore du tube digestif et donner après hydrolyse des composés toxiques capables de provoquer un dérèglement important du métabolisme dans de nombreux organes tels que les reins, la thyroidde, le foie ou les testicules, avec des répercussions sur la croissance, la production et la reproduction. Il en résulte une diminution des performances zootechniques.

L'objectif de la présente étude est d'identifier les principes antinutritionnels et leurs métabolites contenus dans du tourteau de colza de la variété samourai (variété 00) obtenu par pres- sion et de mesurer l'évolution des concentrations en fonction du temps de séjour dans le rumen et en fonction du taux d'incorporation du tourteau de colza dans la ration d'engraissement donnée à des taurillons.

\section{MATÉRIEL ET MÉTHODES}

\section{Animaux}

Huit taurillons blanc bleu belge du type mixte et d'un poids moyen de $530 \mathrm{~kg}$ ont été utilisés dans un modèle de deux carrés latins $4 \times 4$. Ils ont été munis d'une canule du rumen et maintenus dans des loges individuelles du type stalle à métabolisme. Ils recevaient une ration d'engraissement à base de pulpes séchées dans laquelle les matières azotées de tourteau de soja et de lin ont été remplacées à raison de 33 (ration $1 / 3 \mathrm{~T}$ colza), 66 (ration $2 / 3 \mathrm{~T}$ colza) et $100 \%$ (ration $3 / 3 \mathrm{~T}$ colza) par une quantité équivalente de matières azotées de tourteau de colza. Le colza utilisé était de la variété Samouraï double 00 . Le schéma expérimental et la composition des rations ont été décrits dans un article précédent (Mayombo et al, 1997).

\section{Mesures et méthodes}

Les méthodes analytiques employées avaient pour but d'identifier et de mesurer les glucosinolates et leurs produits de dégradation dans le tourteau utilisé pour la préparation des rations. 
Elles ont permis également d'évaluer l'influence du temps de séjour dans le rumen sur la concentration de ces facteurs antinutritionnels dans des résidus humides de tourteau de colza ou dans le liquide et contenu du rumen.

\section{Résidus humides de tourteau de colza}

L'identification et la mesure des glucosinolates et de leur dérivés dans le tourteau et dans les résidus ont été réalisées grâce à la technique des sachets de nylon. Pour ce type d'étude, l'influence de la ration peut avoir une importance. Néanmoins, en vue de réduire le nombre d'échantillons, seuls les animaux recevant les rations $1 / 3 \mathrm{~T}$ colza et $2 / 3 \mathrm{~T}$ colza - soit les niveaux d'incorporation intermédiaires - ont été utilisés. Des sachets de nylon vides d'une taille de $12 \times 7,5 \mathrm{~cm}$ ont été remplis avec $6 \mathrm{~g}$ de tourteau de colza. Ils ont été attachés à des tuyaux en plastique portant chacun cinq sachets et ont ensuite été introduits dans le rumen des animaux. Les sachets de nylon ont été retirés par série de deux après une durée d'incubation d' $1,2,3,4,5,6$, $7,8,9$ et 10 heures. Les mesures ont été répétées quatre fois, et les échantillons regroupés pour les dosages.

\section{Prélèvement du liquide et du contenu du rumen}

L'étude cinétique de la « dégradation » des principes antinutritionnels dans le rumen a été réalisée sur la phase liquide et la phase solide du contenu du rumen. Les prélèvements ont été réalisés sur tous les animaux. Le matériel utilisé a consisté en une sonde en plastique semi-rigide de diamètre de $2,5 \mathrm{~cm}$, d'un récipient pour l'aspiration et la récolte du contenu du rumen et d'une petite pompe à vide portative. Au cours du prélèvement, la sonde a été constamment déplacée dans la masse du contenu du rumen afin d'assurer une prise d'échantillon aussi représentative que possible du contenu total du rumen. Les prélèvements ont été opérés avant le repas du matin et $1,2,3$ et 4 heures après ce repas. Du thiomersal à $3 \%$ a été ajouté et mélangé au contenu du rumen à raison de $2 \mathrm{~mL}$ pour $60 \mathrm{~mL}$ de contenu. La partie solide a été séparée de la partie liquide par filtration sur de la gaze. Après séparation, les échantillons ont été congelés en vue d'analyses chimiques.

\section{Analyses chimiques}

Après une purification sur une colonne échangeuse d'ions (DEAE sephadex A25) et réaction enzymatique avec la sulfatase d'Helix pomatia, les glucosinolates ont été dosés individuellement par HPLC sous forme de désulfoglucosinolates (méthode officielle ISO 9167-1).

L'extraction des produits de dégradation (isothiocyanates, nitriles et vinyl oxazolidine thione) a été réalisée à l'éther diéthylique. Leur séparation, leur identification et leur dosage ont été effectués par chromatographie en phase gazeuse (Hewlett Packard 5890 série II +) couplée à la spectrométrie de masse (détecteur de masse 5972 A). La colonne chromatographique était une HP5 MS (Cat : HP part $n^{\circ} 19091$ S-433), $5 \%$ phényl méthyl silicone.

Les identifications ont été réalisées par GCMS en utilisant deux banques de spectres de masse de référence (WILEY 138 et NBS $75 \mathrm{~K}$ ) et par l'analyse des ions caractéristiques.

\section{RÉSULTATS}

\section{Glucosinolates et leurs produits de dégradation dans le tourteau}

Les teneurs individuelles du tourteau de colza de la variété Samouraï en glucosinolates sont reprises au tableau I. La concentration totale en glucosinolates a été de $17,6 \mu \mathrm{mol} / \mathrm{g}$ de matière sèche (MS) de tourteau. Les glucosinolates les plus représentés étaient la progoitrine, la gluconapine et la 4-OH glucobrassicine. La disparition des glucosinolates a été relativement rapide (tableau II). Après 4 ou 5 heures d'incubation, le résidu humide restant dans les sachets ne contenait plus de glucosinolates. Par rapport aux concentrations observées dans le tourteau et en comparant avec les résultats obtenus après une 
Tableau I. Composition et teneur en glucosinolates dans le tourteau $(\mu \mathrm{mol} / \mathrm{g} \mathrm{MS})$.

\begin{tabular}{lc}
$-1 \cdots$ & Teneurs \\
Glucosinolates & $-\cdots, 22$ \\
\hline Progoitrine & 0,44 \\
Gluconapoléiférine & 3,73 \\
Gluconapine & 2,11 \\
4-OH glucobrassicine & 1,05 \\
Glucobrassicanapine & 0,23 \\
Glucobrassicine & 0,79 \\
Gluconasturtine & 17,57 \\
Total &
\end{tabular}

heure d'incubation, il semble que c'est la progoitrine, un glucosinolate hydroxylé, qui ait disparu le plus rapidement. Il est intéressant également de noter que la disparition a été moins rapide (des résidus étaient encore présents après 4 heures) et moins intense (concentration plus élevée) lorsque la ration contenait plus de tourteau de colza.

Parmi les produits habituels de dégradation des glucosinolates, seul le 3-hydroxypent-4-ènenitrile a été détecté dans le tourteau initial (7,2 $\mu \mathrm{g} / \mathrm{g}$ MS). Dans les résidus, le 3-hydroxypent-4-ènenitrile et la 5-vinyl-1, 3-oxazolidine2-thione, deux composés pouvant provenir de la progoitrine, ont été identifiés ainsi que le but3-ènylisothiocyanate venant de la gluconapine. Un autre nitrile, le 3-phénylpropane nitrile, a

Tableau II. Teneurs en glucosinolates ( $\mu \mathrm{mol} / \mathrm{g}$ MS) dans les résidus humides du tourteau de colza incubé dans le rumen de taurillons nourris avec des rations dont 33 et $66 \%$ des matières azotées provenant des tourteaux étaient des matières azotées de tourteau de colza.

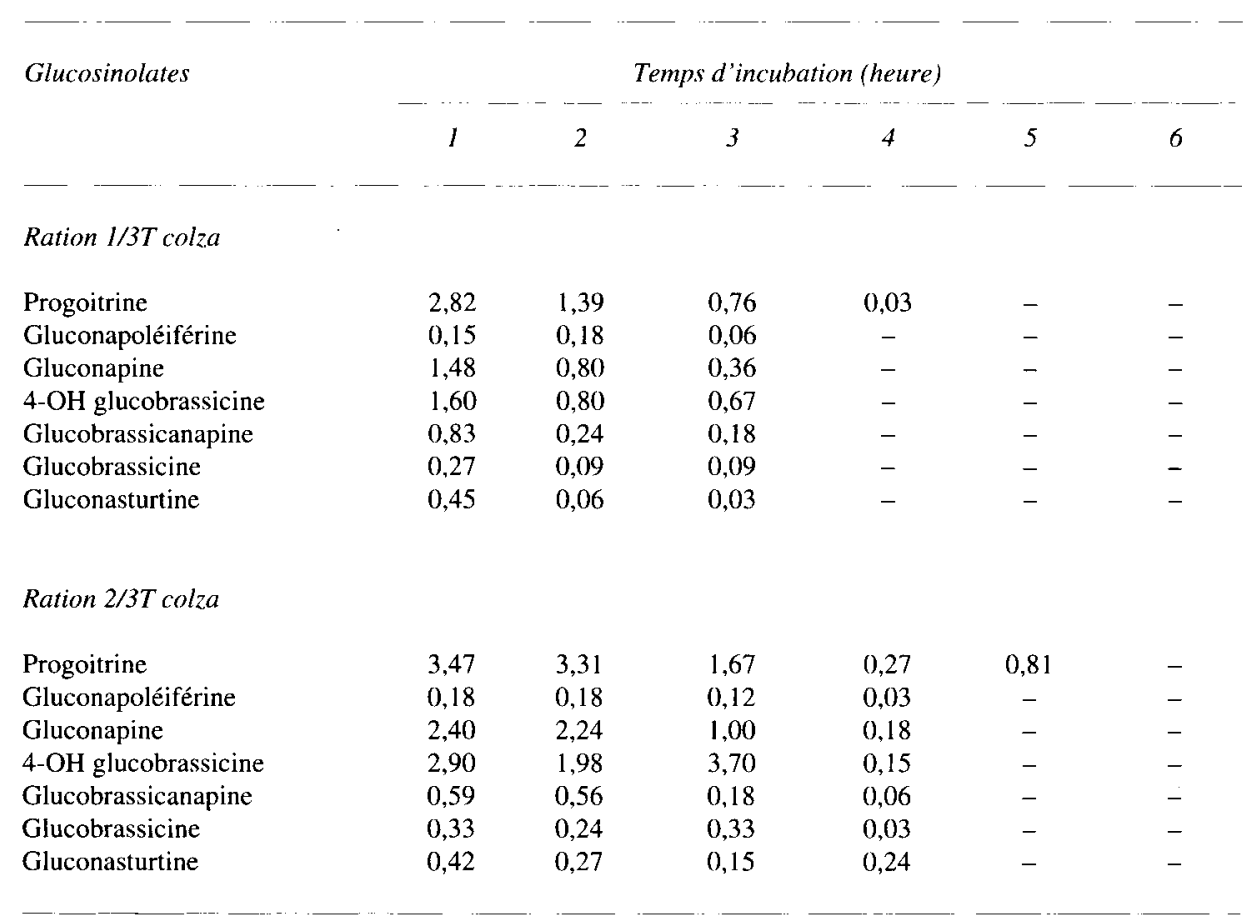

- : Valeur en dessous de la limite de sensibilité de la méthode 
Tableau III. Teneurs en produits de dégradation dans les résidus humides du tourteau de colza incubé dans le rumen de taurillons nourris avec des rations dont 33 et $66 \%$ des matières azotées provenant des tourteaux étaient des matières azotées de tourteau de colza $(\mu \mathrm{g} / \mathrm{g} \mathrm{MS})$.

\begin{tabular}{|c|c|c|c|c|c|c|}
\hline \multirow{3}{*}{$\begin{array}{l}\text { Composés } \\
\qquad-\ldots-------\end{array}$} & \multicolumn{6}{|c|}{ Temps d'incubation (heure) } \\
\hline & 1 & 2 & 3 & 4 & 5 & 6 \\
\hline & - & - & - & $-\quad \cdot-$ & & $--\quad-$ \\
\hline \multicolumn{7}{|l|}{ Ration $1 / 3 T$ colza } \\
\hline \multicolumn{7}{|l|}{ Progoitrine } \\
\hline 3-hydroxypent-4-ènenitrile & 25,52 & 11,98 & 2,73 & 5,44 & - & - \\
\hline 5-vinyl-1, 3-oxazolidone-2-thione & 16,02 & 9,00 & 5,07 & 4,19 & - & - \\
\hline \multicolumn{7}{|l|}{ Gluconapine } \\
\hline but-3-énylisothiocyanate & 3,53 & 1,42 & 1,70 & - & - & - \\
\hline 3-phénylpropanenitrile & 5,73 & 2,72 & 1,12 & - & & 1,53 \\
\hline 3-phénylpropanoique & 91,99 & 40,44 & 28,22 & 55,80 & 30,14 & 125,44 \\
\hline 2-phényléthanoïque & 14,45 & 5,40 & 3,31 & 4,07 & 2,62 & 12,66 \\
\hline 3-méthylindole & 2,76 & 1,42 & 1,06 & 3,98 & 2,42 & 6,26 \\
\hline
\end{tabular}

\section{Ration 2/3T colza}

\section{Progoitrine}

3-hydroxypent-4-ènenitrile

5-vinyl-1, 3-oxazolidone-2-thione

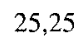

18,72

14,58

15,61

3,98

6,08

60,62

7,51

2,11
Gluconapine

but-3-ènylisothiocyanate

3-phénylpropanenitrile

3-phénylpropanoíque

2-phényléthanoïque

3-méthylindole

$\begin{array}{llll}3,07 & 3,55 & 5,56 & 0,61\end{array}$

$4,01 \quad 4,28$

$84,77 \quad 93,47$

$6,85 \quad 7,47$

5,26
4,65

3,65

5,56

2,67

42,56

$-$

6,65
0,52

2,36

0,61

1,16

119,86

4,20

7,69

\section{8,57}

$-$

4,80

_ : Valeur en dessous de la limite de sensibilité de la méthode

été également identifié mais il ne peut, en principe, être synthétisé à partir des glucosinolates contenus dans le tourteau de colza. À côté des acides volatils ou, gras classiques, deux acides aromatiques acides le 3-phénylpropanoïque, et le 2-phényléthanoïque et le 3-méthylindole ont été aussi détectés (tableau III). Après 1 heure d'incubation, les concentrations en 3-hydroxypent-4-ènenitrile, en 5-vinyl-1,3-oxazolidine2-thione, en but-3-ènylisothiocyanate et en 3- phénylpropanenitrile ont été respectivement de l'ordre de $25,17,4$ et $6 \mu \mathrm{g} / \mathrm{g}$ MS quelle que soit la ration (1/3 $\mathrm{T}$ colza $-2 / 3 \mathrm{~T}$ colza). Les teneurs en nitriles et isothiocyanates sont devenues nulles après 5 à 6 heures d'incubation dans le rumen des taurillons. En revanche, les acides 2-phényléthanoïque ou 3-phénylpropanoïque et le 3-méthylindole sont restés présents dans tous les résidus, y compris après 10 heures d'incubation. 


\section{Liquides et contenus du rumen}

Malgré la très bonne limite de sensibilité de la méthode à environ $10 \mathrm{nmol} / \mathrm{g}$, aucun glucosinolate n'a été mis en évidence dans les liquides et contenus du rumen pour les rations $2 / 3$ et 3/3 T colza. Dans des conditions opératoires identiques à celles utilisées pour les résidus humides (GC-MS avec " scan " complet), aucune trace de nitriles, d'isothiocyanates et de 5-vinyl-1, 3-oxazolidine-2-thione n'a été retrouvée dans les liquides et contenus du rumen analysés pour des rations contenant respectivement 66 et $100 \%$ des matières azotées de tourteau de colza. Afin d'augmenter la sensibilité de la méthode d'un facteur d'environ 50, une recherche par ions a été effectuée. Malgré l'augmentation considérable de la limite de détection des composés (environ $0,02 \mathrm{nmol} / \mathrm{g}$ ), aucune trace de nitriles, isothiocyanates et 5-vinyl-1,3-oxazoli- done-2-thione n'a été mise en évidence dans les échantillons. Ces différents produits n'étant pas identifiés dans les rations riches en colza, les échantillons obtenus avec la ration 1/3 $\mathrm{T}$ colza n'ont pas été analysés. Les liquides et contenus du rumen de la ration témoin ont été toutefois analysés en tant que « Blanco ».

Lors de l'analyse des résidus humides de tourteau de colza, nous avions détecté par spectrométrie de masse la présence d'acide 2-phényléthanoïque, d'acide 3-phénylpropanoïque et du 3-méthylindole (tableau III). Ces trois composés ont à nouveau été identifiés (tableau IV) dans le liquide du rumen, ainsi que dans le contenu du rumen. Il faut noter que les concentrations observées exprimées en MS ont été plus élevées dans le contenu que dans le liquide. Peu de différences ont été enregistrées entre les rations $\mathrm{T}$ colza $2 / 3$ et $\mathrm{T}$ colza $3 / 3$. Il semble que les concentrations aient été plus importantes dans

Tableau IV. Teneur en produits de dégradation dans le liquide et le contenu du rumen ( $\mu \mathrm{g} / \mathrm{g}$ de MS).

\begin{tabular}{|c|c|c|c|c|c|c|c|c|c|}
\hline & \multicolumn{3}{|c|}{ 2-phényléthanoïque } & \multicolumn{3}{|c|}{ 3-phénylpropanö̈que } & \multicolumn{3}{|c|}{ 3-méthylindole } \\
\hline & 0 & $2 / 3$ & $3 / 3$ & 0 & $2 / 3$ & $3 / 3$ & $o$ & $2 / 3$ & $3 / 3$ \\
\hline \multicolumn{10}{|c|}{ Liquide du rumen } \\
\hline $0^{\mathrm{a}}$ & - & - & - & - & - & - & - & 1,12 & 0,68 \\
\hline 1 & - & 1,21 & - & - & 10,07 & 10,59 & - & 1,14 & 0,94 \\
\hline 2 & - & 1,42 & 1,60 & 3,32 & 17,72 & 22,31 & 1,14 & 1,48 & 1,15 \\
\hline 3 & - & 2,99 & 1,09 & 4,11 & 31,73 & 29,13 & 1,12 & 1,42 & 0,97 \\
\hline 4 & - & 1,83 & 0,58 & 5,60 & 33,44 & 29,07 & 0,98 & 1,29 & 0,81 \\
\hline
\end{tabular}

Contenu du rumen

\begin{tabular}{|c|c|c|c|c|c|c|c|c|c|}
\hline $0 *$ & - & - & - & - & - & - & 3,87 & 2,98 & 5,60 \\
\hline 1 & 3,24 & 6,28 & 5,61 & 7,10 & 18,77 & 16,87 & 4,54 & 2,03 & 1,15 \\
\hline 2 & 5,12 & 8,86 & 5,07 & 12,20 & 30,03 & 27,10 & 17,02 & 7,16 & 2,33 \\
\hline 3 & 1,93 & 3,48 & 1,87 & 13,11 & 39,13 & 31,43 & 3,83 & 3,81 & 3,00 \\
\hline 4 & 1,56 & 0,07 & $1,5 \mathrm{I}$ & 13,34 & 29,49 & 39,04 & 3,45 & 2,76 & 7,65 \\
\hline
\end{tabular}

- : Valeur en dessous de la limite de sensibilité de la méthode; is se refère à l'intervalle de temps (exprimé en heure) après le repas du matin. $0:$ ration $0 \mathrm{~T}$ colza $; 2 / 3:$ ration $2 / 3 \mathrm{~T}$ colza $; 3 / 3:$ ration $3 / 3 \mathrm{~T}$ colza. 
les prélèvements effectués plus de 2 heures après la distribution du repas. Enfin, il faut signaler que ces produits ont été isolés également dans le rumen des animaux qui n'avaient pas reçu de tourteau de colza, généralement en concentration plus faible, sauf en ce qui concerne le 3 méthylindole.

\section{DISCUSSION}

La concentration en glucosinolates totaux de tourteau de colza étudié a été de 17,6 $\mu \mathrm{mol} / \mathrm{g}$ de MS, valeur considérée comme faible pour un tourteau de colza du type 00. En effet, la limite maximale pour les variétés de type 00 est fixée entre 20 à $35 \mu \mathrm{mol} / \mathrm{g}$ de MS tandis que dans les variétés de type 0 , la teneur est supérieure à $40 \mu \mathrm{mol} / \mathrm{g}$ de MS (Cooke et al, 1987 ; Schnug, 1987 ; Merrien et Ribaillier, 1988 cités par Merrien, 1993). Les effets négatifs asssociés au tourteau de colza résultent principalement des produits de dégradation des glucosinolates. Cette hydrolyse peut s'effectuer lors du processus de fabrication en particulier lorsqu'une phase de chauffage ou l'utilisation d'un solvant est réalisée (Hill, 1979). Les principaux effets sont situés au niveau de l'appétence et dans le fonctionnement des organes tels que thyroïde, rein et foie. Les résultats du tableau II indiquent que les glucosinolates disparaissent rapidement du tourteau puisqu'après 3 ou 4 heures d'incubation ils ne sont plus détectables. La disparition a été fonction de la proportion de tourteau de colza incorporé dans la ration, les concentrations résiduelles étant plus élevées et maintenues pendant plus longtemps avec la ration 2/3 $\mathrm{T}$ colza. Ce sont les microorganismes du gros intestin (cæcum de volaille, Marangos et Hill, 1974) ou du rumen (Duncan et Milne, 1992) qui sont responsables de la dégradation des glucosinolates et de leurs métabolites. D'après ces derniers auteurs (et du moins en ce qui concerne les molécules de la famille des allyl cyanides), cette activité est adaptative, c'est-à-dire qu'elle est plus importante lorsque l'aliment susceptible de contenir les glucosinolates est présent dans la ration et donc dans le rumen. Cette activité semble avoir été particulièrement importante dans l'expérience présente puisqu'avec une technique de dosage très sensible aucun glucosinolate n'a été mis en évidence, dans le liquide ou dans le contenu de rumen. D'autre part, les produits de dégradation avaient en majorité disparu après 6 heures. Chez les animaux recevant les rations contenant les différentes proportions de tourteau de colza, de nombreuses molécules susceptibles d'être des métabolites des glucosinolates ont été identifiées dans les résidus des sachets de nylon (tableau III) ou dans le liquide et le contenu de rumen (tableau IV). Ces molécules, dont la structure chimique est bien définie, sont assez mal connues concernant leur toxicité. Pour certaines d'entre elles, la DL 50, c'est-à-dire la dose de composés purs qui tue $50 \%$ des animaux testés - en l'occurence des rats -, est connue. D'après Van Etten et al (1969), les nitriles, par exemple le 3-hydroxypent-4-ènenitrile ou le 3phenylpropanènitrile, sont 8 fois plus toxiques que le 5-vinyl-1-3-oxazolidone-2-thione. Les DL 50 sont respectivement de 170 à $240 \mathrm{mg} / \mathrm{kg}$ et de 1260 à $1415 \mathrm{mg} / \mathrm{kg}$. Il faut noter que des substances dont la DL 50 est supérieure à $1000 \mathrm{mg} / \mathrm{kg}$ sont considérées comme non toxiques. Sur la base d'une teneur en nitrile d'environ $30 \mu \mathrm{g} / \mathrm{g}$ de MS dans les résidus après 1 heure d'incubation du tourteau de colza (tableau III), il faudrait l'équivalent d'environ 6 à $7 \mathrm{~kg}$ de MS de résidu de tourteau de colza pour obtenir l'équivalent de la DL $50 \mathrm{de} 200 \mathrm{mg} / \mathrm{kg}$. Une telle quantité de tourteau de colza est totalement irréaliste dans une ration normalement constituée, le maximum incorporé ayant été de $20 \%$ dans la ration 3/3 T colza (Mayombo et al, 1997). Une telle interprétation semble donc indiquer qu'avec le tourteau de colza étudié les principes antinutrionnels tels que les glucosinolates et leur métabolites disparaissent rapidement. Dans ces conditions, les animaux n'ont pas développé de problèmes de santé. C'est vraisemblablement la même interprétation qu'il faut donner pour le peu d'effets sur les ingestions (Mayombo et al, 1997). Dans la revue rapportée par Hill (1991), lorsque le tourteau de colza contenait de faibles teneurs en glucosinolates totaux, aucun effet n'était rapporté sur les ingestions. En revanche, 
dans les essais contenant plus de $100 \mu \mathrm{mol} / \mathrm{g}$ de glucosinolates, des diminutions d'ingestion ont été rapportées (Papas et al, 1979 ; Fiems et Buysse, 1985 ; Hill et al, 1990 ; Lardy et Kerley 1994). Chez les jeunes animaux, les concentrations élevées en glucosinolates peuvent affecter sévèrement les ingestions et les performances (Mawson et al, 1993). Les auteurs rapportent aussi que la palatabilité peut être considérablement améliorée avec un tourteau de colza à faible ou à très faible teneur en glucosinolates (10-30 ou $1-5 \mu \mathrm{mol} / \mathrm{g}$ ). De tels tourteaux peuvent être utilisés jusqu'à $20 \%$ dans des rations pour jeunes bovins et $30 \%$ chez les vaches laitières. Dans une autre étude, Mawson et al (1994) rapportent le manque d'effets négatifs avec un tourteau de colza à $7,7 \mu \mathrm{mol} / \mathrm{g}$ distribué aux jeunes bovins et aux vaches laitières. Les teneurs des produits de dégradation enregistrées dans la présente étude ont été plus faibles que celles rapportées par Fauduet et al (1995) et Huang et al (1995), soit respectivement des teneurs de 50,6 et $116 \mu \mathrm{mol} / \mathrm{g}$ MS. Enfin, la teneur en glucosinolates peut influencer les caractéristiques de dégradabilité. D'après Subuh et al (1994), un tourteau à faible teneur $(25 \mu \mathrm{mol} / \mathrm{g})$ est moins dégradable dans le rumen qu'un tourteau à haute teneur $(92 \mu \mathrm{mol} / \mathrm{g})$.

\section{CONCLUSION}

En conclusion, il apparaît que le tourteau de colza utilisé contenait de faibles teneurs en glucosinolates. Ces derniers ont été rapidement dégradés dans le rumen en différents produits qui, en majorité, ont disparu assez rapidement de l'aliment ainsi que du liquide et du contenu du rumen. Une telle situation est compatible avec l'ingestion et la digestion élevées observées dans l'essai zootechnique.

\section{REMERCIEMENTS}

Les auteurs remercient la Commission des Communautés européennes, et le ministère de l'Agriculture de la région wallonne.

\section{RÉFÉRENCES}

Astwood EB, Greer MA, Ettlinger MG (1949) L-5vinyl-2-thioxazolidone, an antithyroid compound from yellow turnip and from Brassica seeds, $J$ Biol Chem 181, 121

Cooke RJ, Kimber DS, Morgan AG (1987) Change in the glucosinolate content of oilseed rape varieties. In : Proc 7th International Rapeseed Congress, 11-14 mai 1987, Pologne, 532-536

Darroch CS, Bell JM, Keith MO (1990) The effects of moist heat and ammonia on the chemical composition and feeding value of extruded canola screenings for mice. Can J Anim Sci 70, 267-277

Duncan AJ, Milne JA (1992) Rumen microbial degradation of allyl cyanide as a possible explanation for the tolerance of sheep to Brassica-derived glucosinolates. J Sci Food Agric 58, 15-19

Évrard J (1991) Nouvelles perspectives d'emploi offertes pour les tourteaux de colza à très faible teneur en glucosinolates. Bull $d u G C I R C 7,107$ 111

Fauduet H, Coic JP, Lessire M, Quinsac A, Ribaillier D, Rollin P (1995) Rapeseed meal upgrading-pilot scale preparation of rapeseed meal materials with high or low glucosinolate contents. Anim Feed Sci Technol 56, 99-109

Fenwick GR, Spinks EA, Wilkinson AP, Heaney RK, Legoy MA (1986) Effects of processing on the antinutrient content of rapeseed. $J$ Sci Food Agric 37, 735-741

Fiems LO, Buysse FX (1985) Review of the use of rapeseed meal as protein source in diets for ruminants. Rev Agric 38, 261-274

Greer MA, Deeney JM (1959) Antithyroid activity elicited by the ingestion of pure progoitrin, a naturally occurring thioglucoside of the turnip family. $J$ Clin Invest 38, 1465-1474

Hill R (1979) A review of the toxic effects of rapeseed meals with observations on meal from improved varieties. $B r$ Vet $J 135,3-16$

Hill R (1991) Rapeseed meal in the diets of ruminants. Nutr Abst Rev 61, 139-155

Hill R, Vincent IC, Thompson J (1990) The effects on feed intake in weaned calves of low glucosinolate rapeseed meal as the sole protein supplement. Anim Prod 50, 58

Huang S, Liang M, Lardy G, Huff HE, Kerley MS, Hsieh F (1995) Extrusion processing of rapeseed meal for reducing glucosinolates. Anim Feed Sci Technol 56, 1-9 
ISO 9167-1 Standard (1992) Graines de colza - Dosage des glucosinolates - Partie 1 : Méthode par chromatographie liquide à haute performance. Norme de l'Organisation internationale de standardisation

Lardy GP, Kerley MS (1994) Effect of increasing the dietary level of rapeseed meal on intake by growing beef steers. J Anim Sci 72, 1936-1942

Mayombo AP, Baldwin P, Wathelet JP, Marlier M, Istasse L (1997) Incorporation de tourteau de colza obtenu par pression dans une ration d'engraissement chez le taurillon. I. Ingestion, digestibilité et fermentation dans le rumen. Ann Zootech 46, 57-70

Marangos A, Hill R (1974) The hydrolysis and absorption of thioglucosides of rapeseed meal. Proc Nutr Soc 33, $90 \mathrm{~A}$

Mawson R, Heaney RK, Zdunczyk Z, Kozlowska H (1993) Rapeseed meal glucosinolates and their antinutritional effects. 2. Flavour and palatability. Nahrung 37, 336-344

Mawson R, Heaney RK, Zdunczyk Z, Kozlowska H (1994) Rapeseed meal glucosinolates and their antinutritional effects. 3. Animal growth and performance. Nahrung 38, 167-177
Merrien A (1993) Glucosinolates chez le colza : cinétique d'accumulation et principaux facteurs de variation. In : Colloque Glucosinolates (10 années de recherche), 14 janvier 1993, Ardon, ProleaCetiom, 31-35

Papas A, Ingalls JR, Campbell LD (1979) Studies on the effects of rapeseed meal on thyroid status of cattle, glucosinolate and iodine content of milk and other parameters. J Nutr 109, 1129 1139

Schnug E (1987) Relations between sulphur supply and glucosinolate content of " 0 " and " 00 " oilseed rape. In: Proc 7th Intern Rapeseed Congress, 11-14 mai 1987, Pologne, 682-686

Subuh AMH, Rowan TG, Lawrence TLJ (1994) Effect of heat or formaldehyde treatment and differences in basal diet on the rumen degradability of protein in soyabean meal and in rapeseed meals of different glucosinolate content. Anim Feed Sci Technol 49, 297-310

Van Etten CH, Daxembichler ME, Wolff IA (1969) Natural glucosinolate (thioglucosides) in foods and feeds. J Agr Food Chem 17, 483-491 